\title{
Risk factors for dislocation after bipolar hemiarthroplasty: a retrospective case-control study of patients with CT data
}

\author{
Tilman Graulich $^{1}\left[\right.$ D $\cdot$ Pascal Graeff $^{1} \cdot$ Ashish Jaiman $^{2} \cdot$ Stine Nicolaides ${ }^{1} \cdot$ Tarek Omar Pacha $^{1} \cdot$ Marcus Örgel $^{1}$. \\ Christian Macke $^{1} \cdot$ Mohamed Omar $^{1} \cdot$ Christian Krettek $^{1} \cdot$ Emmanouil Liodakis $^{1}$
}

Received: 1 June 2020 / Accepted: 15 October 2020 / Published online: 23 October 2020

(c) The Author(s) 2020

\begin{abstract}
Purpose Bipolar hemiarthroplasty has been shown to have a lower rate of dislocation than total hip arthroplasty. However, as the influencing risk factors for bipolar hemiarthroplasty dislocation remain unclear, we aimed to analyse patient and surgeon-specific influencing risk factors for bipolar hemiarthroplasty dislocation.

Methods We retrospectively analysed patients who were operated between 2012 and 2018 and had dislocated bipolar hemiarthroplasty and matched them to patients without a dislocated bipolar hemiarthroplasty, operated between 2018 and 2019. The study was limited to patients who received either a pre- or postoperative pelvic computed tomography. Besides demographic, morphologic, and physiologic data, we analysed duration of surgery; ASA score; Charlson Comorbidity Index; Almelo Hip Fracture Score; Parker Score; and acetabular morphology angles including acetabular anteversion angle, posterior acetabular sector angle, posterior wall angle, and acetabular roofing.

Results We included nine patients with a dislocated bipolar hemiarthroplasty and 30 with a non-dislocated bipolar hemiarthroplasty. Patient-specific factors prompting a higher risk for dislocated bipolar hemiarthroplasty were longer duration of surgery $(\min )(115 \pm 50$ vs. $80 \pm 27, p=0.01)$; dementia ( $56 \%$ vs. $13 \%, p<0.01)$; smaller posterior acetabular sector angle $\left(^{\circ}\right)(96 \pm 6$ vs. $109 \pm 10, p<0.01)$; and smaller posterior wall angle $\left(^{\circ}\right)(67 \pm 6$ vs. $77 \pm 10, p=0.02)$.

Conclusion Dementia and insufficient posterior wall angle were associated with higher risk of dislocation in bipolar hemiarthroplasty
\end{abstract}

Keywords Hemiarthroplasty $\cdot$ Hip dislocation $\cdot$ Hip arthroplasty $\cdot$ Femoral neck fracture $\cdot$ Posterior acetabular sector angle

Tilman Graulich

graulich.tilman@mh-hannover.de

Pascal Graeff

graeff.pascal@mh-hannover.de

Ashish Jaiman

drashishjaiman@gmail.com

Stine Nicolaides

nicolaides@web.de

Tarek Omar Pacha

omarpacha.tarek@mh-hannover.de

Marcus Örgel

oergel.marcus@mh-hannover.de

Christian Macke

macke.christian@mh-hannover.de
Mohamed Omar

omar.mohamed@mh-hannover.de

Christian Krettek

krettek.christian@mh-hannover.de

Emmanouil Liodakis

liodakis.emmanouil@mh-hannover.de

1 Trauma Department, Hannover Medical School, Carl-Neuberg-Straße 1, 30625 Hannover, Germany

2 Vardhman Mahavir Medical College and Safdarjung Hospital, New Delhi, India 


\section{Introduction}

Classical indications for bipolar hemiarthroplasty (BHA) are displaced femoral neck fractures (Garden types III and IV) in elderly low-demand patients [1]. When measured using the Harris Hip Score, functional results of HA are inferior as compared to total hip arthroplasty (THA) [2]. However, it has also been reported that with BHA, dislocation rates are significantly lower during the first postoperative year, as bipolar cups are larger and have increased jump distance [3]. Nevertheless, dislocations are still a major concern [1]. Hemiarthroplasty is usually recommended for patients $>80$ years or those that have a predicted life expectancy of $<4$ years [1, 4]. Dislocation after THA has been analysed thoroughly and is described to have an incidence of $0.5-3 \%$ [5]. The underlying causes of dislocation after THA have been classified by Wera et al. [4] into the following six categories: (1) acetabular component malpositioning, (2) femoral component malpositioning, (3) abductor deficiency, (4) impingement, (5) poly wear, and (6) unrecognized aetiology (idiopathic). Instability has been reported as the most common reason for revision surgery after THA in the USA [6]. Factors associated with higher dislocation rates post-THA are small head size; femoral component malpositioning; cup inclination outside Lewinnek`s safe zone $\left(30^{\circ}-50^{\circ}\right)$; inappropriate anteversion; unrepaired joint capsule; lowvolume surgeon; and patient-specific factors such as neurological deficits (dementia, Parkinson disease), high ASA score, history of spinal fusion, abductor deficiency, body mass index $>35 \mathrm{~kg} / \mathrm{m}^{2}$, and preoperative Harris Hip Score $<41$ [5, 7-11]. Despite lower dislocation rates, BHA is associated with higher periprosthetic fracture rates most likely because of lower bone quality $[1,3,4,8,12-14]$. Owing to higher patients' satisfaction with THA than HA in case of displaced femoral neck fractures, the former is recommended in patients aged $<80$ years and with a life expectancy of $>4$ years. In patients aged $>80$ years, both methods show equal results [1, 25, 4]. Age $<80$ years is associated with higher dislocation rates than age $>80$ years after HA [4].

Dislocation after BHA seems to be independent from the surgical approach [15]. A recent study has reported that posterior wall acetabular morphology may influence hip stability [16].

Therefore, we aimed to comprehensively describe risk factors for BHA dislocation and attempted to answer the following questions:

1. Are neurological deficits and a higher comorbidity index (i.e. ASA score) associated with higher dislocation rates?
2. Does a larger femoral head size reduce the risk of dislocation?

3. Is posterior acetabular wall morphology an independent risk factor for dislocation?

4. Are there any other factors associated with high dislocation rates?

\section{Methods}

We retrospectively analysed all patients with BHA dislocation that were operated in our institution for femoral neck fracture between 2012 and 2019. The inclusion criteria were posterior dislocated BHA and pelvic CT results. Patients who did not undergo a CT scan were excluded from the study. One patient who was $<18$ years and operated for tumour resection was also excluded. Finally, 9 patients with BHA dislocation met our inclusion criteria. These patients were matched to 30 patients that did not experience dislocation and who were treated between 2018 and 2019 with matching surgical technique and also had pelvic $\mathrm{CT}$ results. All patients were operated by skilled orthopaedic surgeons in the presence of a fellowship-trained orthopaedic trauma surgery consultant. Utmost care was given to capsular closure. A case-control ratio of 1:3 was assumed sufficient for detection of significant differences. All CTs were performed using a Somatom Force (Siemens Healthcare GmbH, Erlangen, Germany). Image slices were 0.6-mm thick. A 3D reconstruction was performed using the Visage 7.1.11 software (Visage Imaging GmbH, Berlin, Germany).

Patient data were collected from our digital patients' records. Demographic and morphologic data such as age, sex, and BMI were obtained. We also recorded comorbidities including dementia and Parkinson disease, ASA score, and neurologic decline, and determined the Charlson Comorbidity Index (CCI) with the estimated 10-year survival (\%), Almelo Hip Fracture Score (AHS) with the predicted risk of early mortality (\%) and the Parker score, a score describing mobility in patients after hip fracture. The cup size, surgical approach, and duration of surgery were the intraoperative factors that were noted. We further determined the kind of infection (superficial/deep) and time since surgery in the case of dislocation.

Polyethylene wear and spino-pelvic imbalance were determined on postoperative radiographs. Radiological evaluation of CT-scans was performed by a trained orthopaedic surgeon (PG), who was blinded to patients' group allocation. Acetabular anteversion angle (AAA), posterior acetabular sector angle (PASA), posterior wall angle (PWA), and acetabular roofing (\%) were evaluated from CT scans. Methodology described by Fullam et al. [15] was used for radiological evaluation. In short, an inter-capital centre line (ICL) was drawn on true axial images through both femoral 
heads at the point of maximum diameter in all 3 planes. An orthogonal line to the ICL-the ICL90 was drawn. A line between the anterior and posterior acetabular lip of the acetabulum was drawn the anteversion line (AVL). The angle between the ICL90 and AVL was measured to determine the AAA. The PASA was determined as described by Valera et al. [17] i.e. by measuring the angle between the ICL and a line from the femoral head centre to the lateral edge of the posterior wall. The PWA was measured by using the angle between the ICL90 and the tangent to the posterior articular surface area. The femoral head coverage/roofing was determined using the ICL and AVL. The part within the acetabulum to the AVL was divided by the whole femoral head diameter to determine the amount of femoral head coverage (\% of total) (Fig. 1).

\section{Statistical analysis}

Data were tested for normal distribution using the Shapiro-Wilk test. For comparative statistics, in case of normal distribution, $t$-test was used. A multivariate ANOVA (MANOVA) was performed. If data were not normally distributed, Mann-Whitney U test was used. Non-normally distributed data are presented as median with interquartile rang, and normally distributed data as mean \pm standard deviation. A post hoc power analysis was performed. A $p$-value $<0.05$ was considered to indicate statistical significance.

\section{Institutional review board approval (IRB approval)}

For this study, IRB approval was not required, because the investigator did not obtain any data through interventional interaction and did not present any identifiable personal information (Fig. 2).

\section{Results}

\section{Demographic, morphologic, and physiologic data}

We included 39 patients (mean, 83 years; range, 64-98 years) in the study. The 9 patients with a dislocated BHA ( $79 \pm 7$ years) did not show significantly difference in age than the 30 patients without dislocated BHA $(84 \pm 7$ years) $(p>0.05)$. No differences were noted between the BMI of both groups ( $22 \pm 3$ vs. $23 \pm 5 ; p>0.05)$. Sex had no influence on dislocation rates $(p>0.05)$. Dementia with a prevalence of $56 \%$ was significantly more common in the
Fig. 1 Radiological measurements: $\mathbf{a}$ in short, an intercapital centre line (ICL) was drawn on true axial images through both femoral heads at the point of the largest diameter in all 3 planes. An orthogonal line to the ICL the ICL90 was drawn. A line between the anterior and posterior acetabular lip of the acetabulum was drawn the anteversion line (AVL). The angle between the ICL90 and AL was measured to determine the AAA. b The femoral head coverage was determined using the ICL and the AVL. The part within the acetabulum to the AVL was divided by the whole femoral head diameter. c The PASA was determined as described by Valera et al. measuring the angle between the ICL and a line from the femoral head centre to the lateral edge of the posterior wall [17]. d The PWA was measured by using the angle between the ICL90 and the tangent to the posterior articular surface area (color figure online)
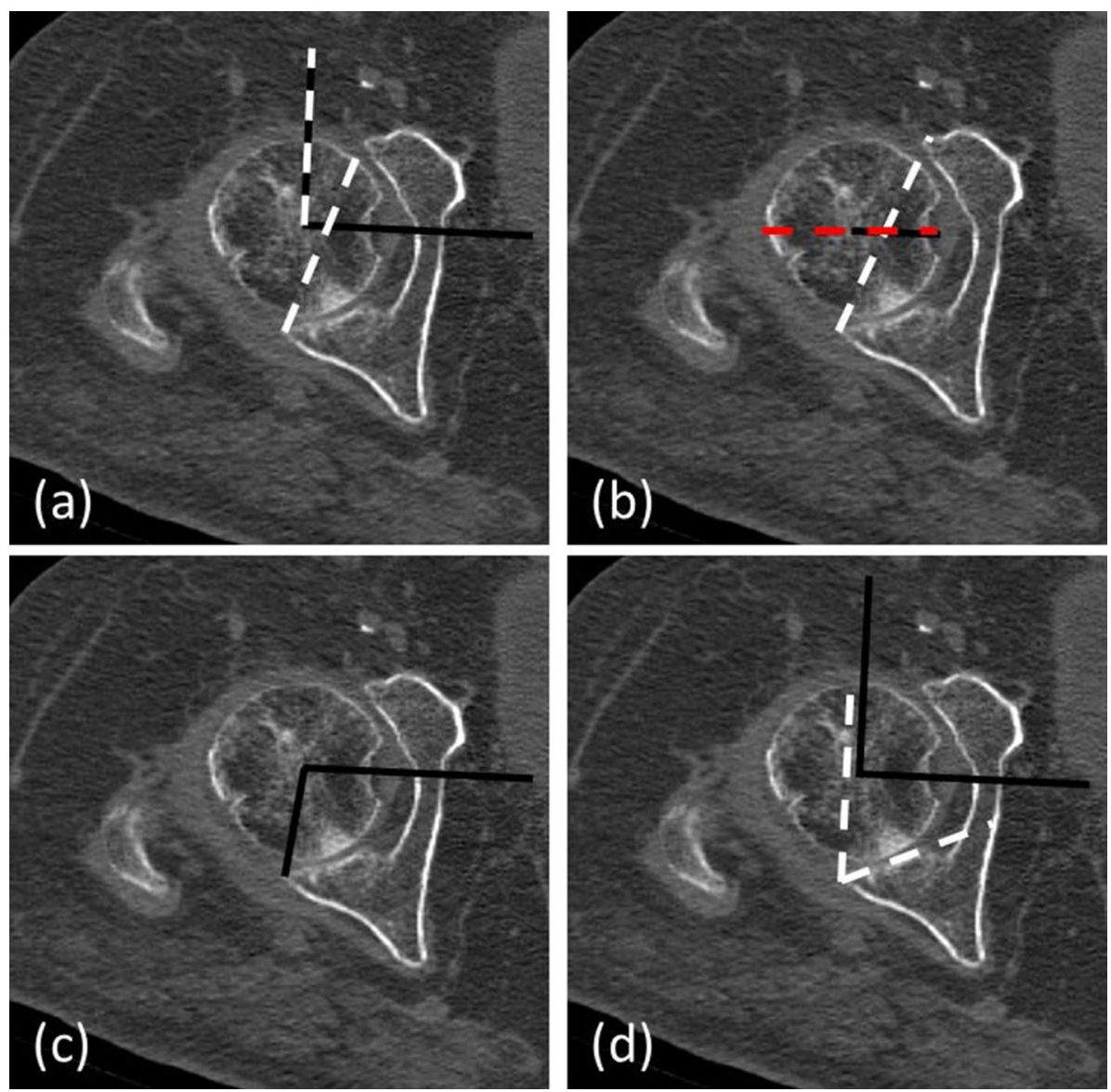


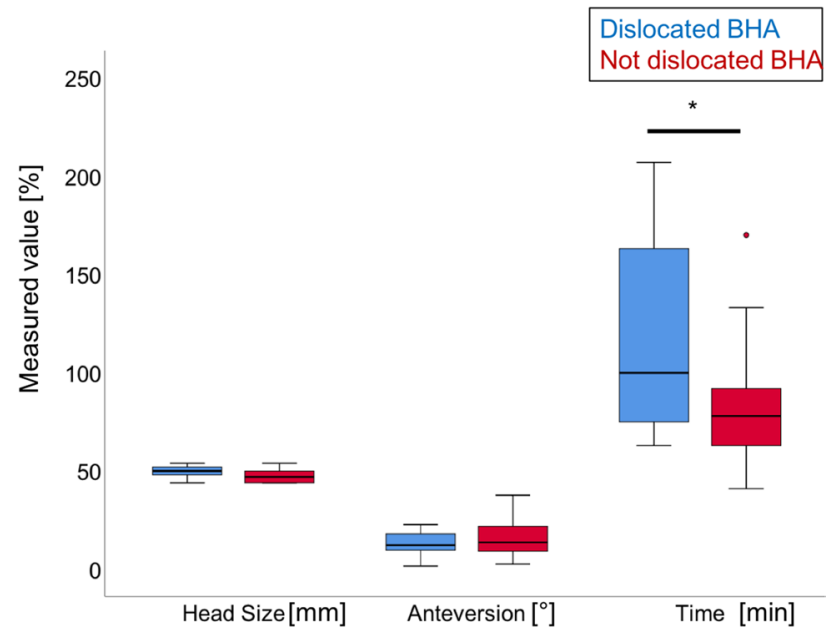

Fig. 2 Univariate Analysis. Comparison of operative parameters between dislocated BHA and non-dislocated BHA Blue: Dislocation group, red: Control group, ${ }^{*}: p<0.05$. Head Size: external head size. Anteversion: femoral shaft anteversion. No differences in femoral shaft anteversion were observed between both groups. Time: operation time. Operation time was longer in patients with dislocated BHA than in patients with non-dislocated BHA (color figure online)

dislocation group than the control group (13\%) $(p=0.009$; OR: 7.6). No patient had Parkinson disease. Infection rates, classified as deep infections, were significantly higher in the dislocation group (40 vs. $0 \% ; p=0.01$ ). The average time to first dislocation was 8 months (range: $0-54$ months) (Table 1).

\section{Comorbidity scores}

Upon comparing the dislocated BHA with the non-dislocated BHA, the ASA score was $2.4 \pm 0.5$ versus $2.7 \pm 0.5$ $(p=0.138), \mathrm{CCI}$ was $6.5 \pm 1.4$ versus $6.2 \pm 2.0(p=0.672)$, estimated survival was $6.9 \pm 9.7$ versus $17.4 \pm 24.2$ $(p=0.192)$. Further, the AHS, Almelo predicted risk, and Parker scores were also not significantly different between both groups ( $p>0.05)$ (Table 1 and Fig. 3).

\section{Radiological measurements}

Most importantly, both PASA and PWA were significantly smaller in the dislocated BHA group than in the non-dislocated controls (PASA: $96 \pm 6$ vs. $109 \pm 10 ; p<0.01$; OR: 12.7; power: $99.6 \%$ ), (PWA: $67 \pm 6$ vs. $77 \pm 10 ; p=0.02$; OR: 7.3 ; power: $97.9 \%$ ). The AAA was not significantly smaller in dislocated BHA than non-dislocated controls $(21 \pm 5$ vs. $25 \pm 6 ; p=0.10$; power: $90 \%)$. The acetabular roofing showed no difference between both groups (Fig. 4 and Table 2). There was no evidence of macroscopic poly wear on radiographs, as the metal head did not show radiographic evidence of eccentricity in the cup. All patients had post-traumatic fracture of the femoral neck without any other systemic musculoskeletal disease, spino-pelvic imbalance, low back pain, or radicular pain.

\section{Operative technique}

Cup size in dislocated BHA was not significantly bigger than in the non-dislocated controls ( $50 \pm 3$ vs. $47 \pm 3 p=0.051$ ). The duration of surgery was significantly longer in patients with dislocated BHA than in the controls $(115 \pm 50$ vs. $80 \pm 27 ; p=0.01$; OR: 4.5 ; power: $79.5 \%$ ) (Fig. 2). Surgical approach showed no differences between both groups, with 7 patients $(78 \%)$ having a lateral approach and two patients (22\%) having a posterior approach in the dislocated BHA group than the $19(63 \%)$ lateral versus $11(37 \%)$ posterior approach in the non-dislocated control group $(p=0.33)$ (Chi-square test).

\begin{tabular}{lllll}
\hline & Total $(n=39)$ & $\begin{array}{l}\text { Dislocated BHA } \\
(n=9)\end{array}$ & $\begin{array}{l}\text { Non-dislocated } \\
\text { BHA }(n=30)\end{array}$ & $p$ value \\
\hline Sex/male (total/\%) & $14 / 35.8$ & $2 / 22$ & $12 / 30$ & 0.448 \\
Age (years) & $81 \pm 13$ & $79 \pm 7$ & $84 \pm 7$ & 0.139 \\
BMI $\left(\mathrm{kg} / \mathrm{m}^{2}\right)$ & $23 \pm 5$ & $22 \pm 4$ & $23 \pm 5$ & 0.634 \\
Dementia (total/\%) & $9 / 23$ & $5 / 56$ & $4 / 13$ & 0.007 \\
Parkinson (total/\%) & $3 / 7.6 \%$ & $0 / 0$ & $3 / 10$ & 0.326 \\
Charlson comorbidity index & $6 \pm 2$ & $6 \pm 1$ & $6 \pm 2$ & 0.635 \\
Estimated survival & $15 \pm 22$ & $7 \pm 10$ & $17 \pm 24$ & 0.240 \\
Almelo hip score & $8 \pm 3$ & $8 \pm 2$ & $8 \pm 3$ & 0.815 \\
Almelo predicted risk & $5 \pm 6$ & $5 \pm 4$ & $6 \pm 6$ & 0.577 \\
Parker score & $6 \pm 2$ & $6 \pm 3$ & $6 \pm 2$ & 0.085 \\
\hline
\end{tabular}




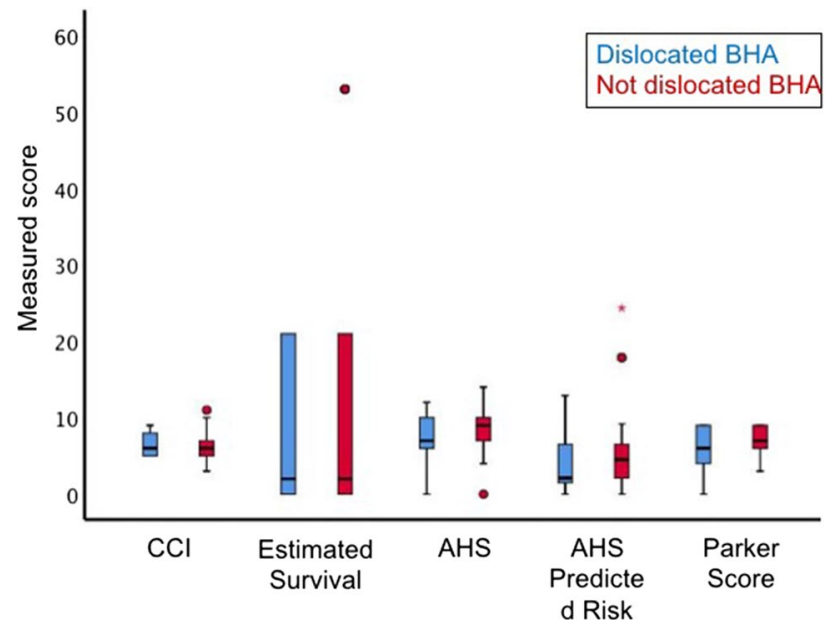

Fig. 3 Comorbidities in the dislocation and control group. Blue: dislocated BHA, red: non-dislocated BHA, CCI: Charlson Comorbidity Index, Estimated survival: Estimated survival based on CCI, AHS: Almelo Hip Score. No differences could be observed between both groups (color figure online)

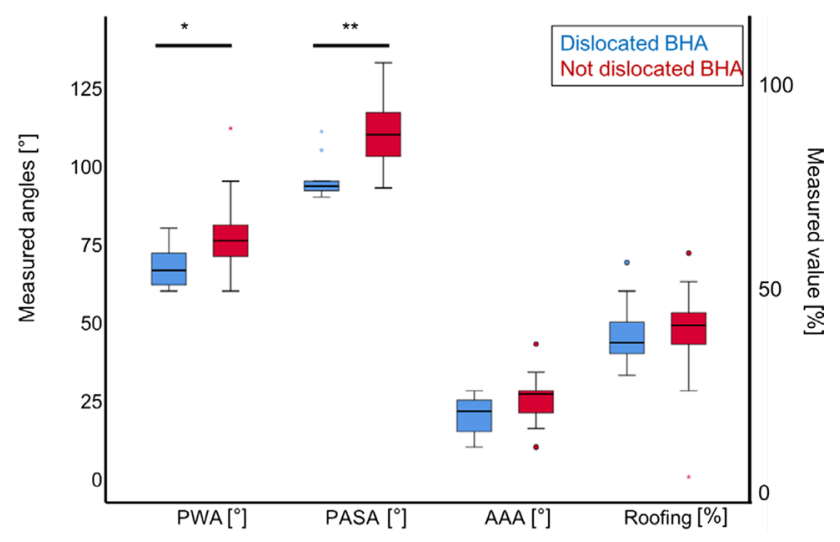

Fig. 4 Univariate Analysis. Comparison of acetabular angles and roofing between dislocated BHA and non-dislocated BHA. Blue: dislocated BHA red: non-dislocated BHA, ${ }^{*} p<0.01,{ }^{*} p<0.05$. PWA: posterior wall angle, PASA: posterior acetabular sector angle, AAA: anteversion acetabular angle, Roofing (\%): Femoral head coverage by the acetabulum. dislocated BHA shows significantly lower values for PWA, PASA, and AAA but no differences in acetabular roofing compared to non-dislocated BHA

Table 2 Radiological measurements

\begin{tabular}{lclll}
\hline & Total $(n=39)$ & $\begin{array}{l}\text { Dislocated } \\
\text { BHA }(n=9)\end{array}$ & $\begin{array}{l}\text { Non-dislocated } \\
\operatorname{BHA}(n=30)\end{array}$ & $p$ value \\
\hline $\begin{array}{l}\text { Acetabular } \\
\text { roofing } \\
(\%)\end{array}$ & $47 \pm 12$ & $47 \pm 11$ & $47 \pm 13$ & 0.939 \\
AAA $\left(^{\circ}\right)$ & $24 \pm 7$ & $19 \pm 5$ & $25 \pm 6$ & 0.105 \\
PASA $\left(^{\circ}\right)$ & $106 \pm 11$ & $96 \pm 6$ & $109 \pm 10$ & 0.001 \\
PWA $\left(^{\circ}\right)$ & $74 \pm 10$ & $66 \pm 6$ & $77 \pm 10$ & 0.02 \\
\hline
\end{tabular}

\section{Discussion}

The present study provides comprehensive information about risk factors associated with BHA dislocation. Our findings revealed that dementia is associated with a risk for dislocation. Further, cup size was not associated with higher dislocation risk. Last, posterior acetabular wall morphology with smaller PASA and PWA were associated with higher dislocation rates.

Neurological deficits are known to negatively influence the postoperative outcome after THA [6, 10, 18, 19]. Interestingly, ASA score, CCI, AHS, and Parker Score did not differ significantly between both groups. However, previously published data have shown a high association of comorbidities with the risk of THA dislocation; contradictory to our expectation, we could not observe these differences in our patients after BHA $[6,10,20]$. We believe that the small sample size is the main reason for not detecting significance of comorbidities.

All patients were operated upon by using a $28-\mathrm{mm}$ internal head and different sizes of external cups (44-54 mm). Our results of smaller cup sizes tending to be associated with less dislocation rates after BHA are contradictory to the literature. To our knowledge, the small sample size is the most likely reason for this discrepancy [21].

Furthermore, longer operation time correlated with a higher dislocation risk. We believe that this is related to less experienced surgeons, which has also been reported in literature that low-volume surgeons show higher complication rates in primary THA and BHA than high-volume surgeons $[10,22]$. Nevertheless, patients with both dislocated BHA and non-dislocated BHA were operated by multiple surgeons (10 vs. 14 surgeons).

Interestingly, as previously shown in the literature, younger patients who were $<80$ years old tended towards higher dislocation rates than those $>80$ years [4]. This might be due to the more active lifestyle of younger patients, or because of less bony coverage owing to fewer posterior osteophytes in younger patients.

It has been shown that the bony acetabular coverage measured by a low centre edge (CE) angle has a strong association for a higher risk for dislocation [18]. Recently Kizkapan et al. [23] described a strong correlation of decreased $\mathrm{CE}$ angle and, more importantly, a reduced femoral offset to be associated with dislocation in BHA. This shows that although operative technique is an important factor, patientspecific anatomy is an equally important and individual factor that can influence the odds for dislocated BHA.

As for most BHA dislocations and as noted in our study, all nine dislocations were posterior dislocations. We believe that a larger bony posterior wall has a high impact and protective function to prevent posterior BHA dislocations. 
Recently, we also described the reference values for the posterior wall coverage with a mean PASA of $100^{\circ}$ and PWA of $72^{\circ}$ in a healthy patient collective [16]. Our data with reduced PASA and PWA in patients with dislocated BHAs (PASA: $96 \pm 6$ vs. $109 \pm 10 ; p<0.01$ ) and (PWA: $67 \pm 6$ vs. $77 \pm 10$; $p=0.02$ ) show that a more open acetabular posterior wall is associated with a higher risk for BHA dislocation.

The debate on influence of surgical approach on dislocation is ongoing. Both the direct lateral approach and posterior approach have been used for BHA, with a tendency towards better functional outcome after the posterior approach; however, it is associated with higher dislocation rates when compared to the lateral approach [15, 24]. In our study, we did not observe significant differences with respect to this aspect of surgery.

As an extrapolation of this study's data, we devised an inhouse algorithm for elderly patients with femoral neck fracture. All patients with a displaced femoral neck fracture (Garden type III or IV) without any clinical sign of hip arthrosis like pre-existing pain should be included and considered as potential candidates for BHA arthroplasty. In the case of dementia, we would either perform a pre-operative CT scan for measurement of acetabular posterior wall indices or subjectively evaluate the posterior wall intraoperatively and label it as closed or open. In the case of a PASA $<95.5^{\circ}$ and $\mathrm{PWA}<71.5^{\circ}$ or an intraoperative open acetabulum, we would consider a dual mobility cup or a THA with a constrained liner, instead of BHA. In the case of no dementia and PASA and PWA $>95.5^{\circ}$ and $71.5^{\circ}$, respectively, or an intraoperative closed acetabulum, we would consider a BHA arthroplasty. Alternatively, in place of measuring the posterior wall angles, the decision can be made by 3D reconstructions as recently described [16].

Our study has some limitations. The retrospective nature is the first limitation of this study, as investigators were required to rely on the availability and accuracy of medical records. Second, no subject in the control group had a torsion difference CT, and only 5 patients in the dislocation group had a torsion difference CT. This means that femoral stem anteversion could not be compared between the 2 groups. Last, the sample size was very small. Although for some parameters like radiological measurements, a high post-hoc power (79.5-99.6\%) could be achieved, we believe that further parameters like comorbidity scores and neurological deficits (e.g. Parkinson's disease) are underpowered (power: 5-51.4\%) and might not reflect the clinical importance of these parameters.

\section{Conclusion}

To the best of our knowledge, this is the first study to evaluate CT data to detect anatomy-associated risk factors for dislocation after BHA for femoral neck fracture.
While many studies concentrated on dislocation risks for THA, this study comprehensively described risk factors for BHA dislocation showing that besides neurological deficits like dementia, the posterior wall anatomy is an important independent factor associated with dislocation rates.

Considering that dislocation after BHA is not so common, further large-sampled, multi-centre, prospective studies should be conducted to validate our results.

Funding Open Access funding enabled and organized by Projekt DEAL.

\section{Compliance with ethical standards}

Conflict of interest The authors declare that there is no conflict of interest.

Open Access This article is licensed under a Creative Commons Attribution 4.0 International License, which permits use, sharing, adaptation, distribution and reproduction in any medium or format, as long as you give appropriate credit to the original author(s) and the source, provide a link to the Creative Commons licence, and indicate if changes were made. The images or other third party material in this article are included in the article's Creative Commons licence, unless indicated otherwise in a credit line to the material. If material is not included in the article's Creative Commons licence and your intended use is not permitted by statutory regulation or exceeds the permitted use, you will need to obtain permission directly from the copyright holder. To view a copy of this licence, visit http://creativecommons.org/licenses/by/4.0/.

\section{References}

1. Lewis DP, Wæver D, Thorninger R, Donnelly WJ (2019) Hemiarthroplasty vs total hip arthroplasty for the management of displaced neck of femur fractures: a systematic review and meta-analysis. J Arthroplast 34(8):1837-1843.e2. https://doi.org/10.1016/j. arth.2019.03.070

2. Yu L, Wang Y, Chen J (2012) Total hip arthroplasty versus hemiarthroplasty for displaced femoral neck fractures: meta-analysis of randomized trials hip. Clin Orthop Relat Res 470(8):2235-2243. https://doi.org/10.1007/s11999-012-2293-8

3. Kreipke R, Rogmark C, Pedersen AB et al (2019) Dual mobility cups: effect on risk of revision of primary total hip arthroplasty due to osteoarthritis - a matched population-based study using the nordic arthroplasty register association database. J Bone Jt Surg Am 101:169-176. https://doi.org/10.2106/JBJS.17.00841

4. Eskildsen SM, Kamath GV, Del Gaizo DJ (2018) Age matters when comparing hemiarthroplasty and total hip arthroplasty for femoral neck fractures in Medicare patients. HIP Int. https://doi. org/10.1177/1120700018816924

5. Herman A, Masri BA, Duncan CP et al (2019) Multivariate analysis of risk factors for re-dislocation after revision for dislocation after total hip arthroplasty. HIP Int. https://doi.org/10.1177/11207 00019831628

6. Jones CW, De Martino I, D'Apolito R et al (2019) The use of dual-mobility bearings in patients at high risk of dislocation. Bone Jt J 101B:41-45. https://doi.org/10.1302/0301-620X.101B1.BJJ2018-0506.R1 
7. Wera GD, Ting NT, Moric M et al (2012) Classification and management of the unstable total hip arthroplasty. J Arthroplast 27:710-715. https://doi.org/10.1016/j.arth.2011.09.010

8. Fessy MH, Putman S, Viste A et al (2017) What are the risk factors for dislocation in primary total hip arthroplasty? A multicenter case-control study of 128 unstable and 438 stable hips. Orthop Traumatol Surg Res 103:663-668. https://doi. org/10.1016/j.otsr.2017.05.014

9. Kurtz SM, Lau EC, Ong KL et al (2017) Which clinical and patient factors influence the national economic burden of hospital readmissions after total joint arthroplasty? Clin Orthop Relat Res 475:2926-2937. https://doi.org/10.1007/s11999-017-5244-6

10. Annan J, Clement N, Macpherson GJ et al (2018) Body mass index, hip function and surgeon volume are independent predictors of dislocation: an analysis of 4334 total hip replacements. Eur J Orthop Surg Traumatol 28:899-905. https://doi.org/10.1007/ s00590-017-2078-6

11. Sadhu A, Nam D, Coobs BR et al (2017) Acetabular component position and the risk of dislocation following primary and revision total hip arthroplasty: a matched cohort analysis. J Arthroplast 32:987-991. https://doi.org/10.1016/j.arth.2016.08.008

12. Moerman S, Mathijssen NMC, Tuinebreijer WE et al (2018) Hemiarthroplasty and total hip arthroplasty in 30,830 patients with hip fractures: data from the Dutch Arthroplasty Register on revision and risk factors for revision. Acta Orthop 89:509-514. https://doi.org/10.1080/17453674.2018.1499069

13. Sappey-Marinier E, Viste A, Blangero Y et al (2019) A comparative study about the incidence of dislocation and peri-prosthetic fracture between dual mobility versus standard cups after primary total hip arthroplasty. Int Orthop. https://doi.org/10.1007/s0026 4-018-4279-y

14. Metcalfe D, Judge A, Perry DC et al (2019) Total hip arthroplasty versus hemiarthroplasty for independently mobile older adults with intracapsular hip fractures. BMC Musculoskelet Dis 4:1-11

15. Fullam J, Theodosi PG, Charity J, Goodwin VA (2019) A scoping review comparing two common surgical approaches to the hip for hemiarthroplasty. BMC Surg 19:1-8. https://doi.org/10.1186/ s12893-019-0493-9

16. Graulich T, Graef P, Nicolaides S et al (2020) Acetabular posterior wall morphology: a CT-based method to distinguish two acetabular posterior wall types. J Orthop 20:160-166. https://doi. org/10.1016/j.jor.2020.01.027
17. Valera M, Ibáñez N, Sancho R et al (2018) Acetabular overcoverage in the horizontal plane: an underdiagnosed trigger of early hip arthritis: a CT scan study in young adults. Arch Orthop Trauma Surg 138:73-82. https://doi.org/10.1007/s00402-017-2811-y

18. Li L, Ren J, Liu J et al (2016) What are the risk factors for dislocation of hip bipolar hemiarthroplasty through the anterolateral approach? A nested case-control study. Clin Orthop Relat Res 474:2622-2629. https://doi.org/10.1007/s11999-016-5053-3

19. Jones C, Briffa N, Jacob J, Hargrove R (2017) The dislocated hip hemiarthroplasty: current concepts of etiological factors and management. Open Orthop J. https://doi.org/10.2174/1874325001 711011200

20. Menendez ME, Ring D, Barnes CL (2016) Inpatient dislocation after primary total hip arthroplasty. J Arthroplast 31:2889-2893. https://doi.org/10.1016/j.arth.2016.05.007

21. Rath B, Eschweiler J, Beckmann J et al (2019) Revisionsendoprothetik der Hüfte Revision total hip arthroplasty. Orthopade 48:315-321. https://doi.org/10.1007/s00132-019-03704-x

22. Clement RC, Strassle PD, Ostrum RF (2018) Should all orthopaedists perform hemiarthroplasty for femoral neck fractures? A volume-outcome analysis. J Orthop Trauma. https://doi.org/10.1097/ BOT.0000000000001176

23. Kizkapan TB, Misir A, Uzun E et al (2020) Factors affecting dislocation after bipolar hemiarthroplasty in patients with femoral neck fracture. Injury. https://doi.org/10.1016/j.injury.2020.01.025

24. Zijlstra WP, De Hartog B, Van Steenbergen LN et al (2017) Effect of femoral head size and surgical approach on risk of revision for dislocation after total hip arthroplasty: an analysis of 166,231 procedures in the Dutch Arthroplasty Register (LROI). Acta Orthop 88:395-401. https://doi.org/10.1080/17453674.2017.1317515

25. Nonne D, Sanna F, Bardelli A et al (2019) Use of a dual mobility cup to prevent hip early arthroplasty dislocation in patients at high falls risk. Injury. https://doi.org/10.1016/j.injury.2019.01.022

Publisher's Note Springer Nature remains neutral with regard to jurisdictional claims in published maps and institutional affiliations. 